\title{
Fuzzy Logic based Health Care System using Wireless Body Area Network
}

\author{
Prakashgoud Patil \\ B.V.B. College of Engineering and Technology \\ Hubli - 580031, Karnataka, India
}

\author{
Samina Mohsin \\ Tontadarya College of Engineering \\ Gadag - 582 101, Karnataka, INDIA
}

\begin{abstract}
Wireless Sensor Networks (WSN) offers proficient communication solution to the ever-present healthcare systems. The miniaturized sensors together with advance micro-electro-mechanical systems (MEMS) technology create a body sensor network (BSN) that continuously monitors the health condition of patients. Some of the other applications where WSN can be used are military, interactive entertainment, and portable audio/video systems. The proposed system for measuring health parameters of patient consists of temperature and pulse sensor connected to base station through a microcontroller and allowing device to be controlled and monitored by remote computer. Wireless sensor network system continuously monitors pulse and temperature of patients at remote or in hospital. A wearable wireless sensor system is designed to continuously capture and transmit the bio-signals to the Doctor / Patient mobile phone. In case of emergency alert will be issued to doctors, relatives, and ambulance in the form of Short Message Services (SMS). Doctor can also provide remote prescription for the patients. Data stored at database is passed to fuzzy logic controller to improve accuracy and amount of data to be sent to the remote user. As the data read from sensor are imprecise / crisp here we design a Fuzzy logic controller (FLC), which is one of component in our computing prototype health status. The FLC system receives context information from sensor as input (which is in crisp form) and the fuzzification module converts input into fuzzy linguistic variable and output is sent to Patient / Doctor which can be easily understood by common person.
\end{abstract}

\section{Keywords}

Fuzzy logic controller, Fuzzifier, Fuzzy Rules, Inference Engine, Wireless Body Area Network (WBAN)

\section{INTRODUCTION}

Over few years WSN have proved their potentiality in various applications. A WSN is a collection of typically small, battery-powered, autonomous, wireless devices (also known as nodes). These devices have on-board processing, communication and sensing capabilities that can monitor physical or environmental conditions, such as temperature, sound, pressure, etc and pass data through a unidirectional or bi-directional network. The nodes are composed of low-power processor that has limited processing, memory device with limited storage capacity, a radio transceiver with a low-power internal/external antenna, low-data rate \& limited range, sensors (scalar, cameras, microphones), and power source (batteries and solar cells).

Each device is normally battery operated. Examining each such single device individually might appear to have small utility.

WSNs find their place strong in the military applications. This extensive usage of WSNs drives into sensor networks research
However, WSNs offer varied applications to most diverse fields like environmental monitoring, habitat monitoring, classroom/home, structural monitoring and health monitoring. Based on their characteristics, each of the applications has its own design concept and implementation to meet its specific requirements [1].

The advances of technology along with WSN's characteristics offer the maximum benefits to health care. A sensor network that is designed to sense the health parameters of a human being is a body sensor network (BSN). The nodes of BSNs are directly attached to human body and this requires utmost care. Some of the health care applications require the BSN to continuously operate gathering patient data for several days without user intervention. Such applications need to take care of the energy constraints of the sensor networks [3].

\section{Challenges in Wireless Body Sensor Networks}

In this modern life health-care is a must for quality life of every individual. The growing population of developed countries and government's budget are directly proportional. This represents challenges for health-care systems. One of the important challenges is to facilitate health-care for the senior citizens living independently. Generally, health monitoring is performed on a periodic check basis, where the patient must remember its symptoms; the doctor performs some tests and plans a diagnostic, then monitors patient progress along the treatment. Healthcare applications of wireless sensor networks allow in-home assistance, smart nursing homes, clinical trial and research augmentation. Before describing medical applications of BSNs, the following section focuses on several challenges and general aspects that describe this kind of technology. Challenges in healthcare application includes: low power, limited computation, security and interference, material constraints, robustness, continuous operation, and regulatory requirements with elderly people [4].

\section{LITERATURE REVIEW}

In this section some related approaches have been discussed. In [3], authors present the system architecture based on a three-tier Body Sensor Network. The BSN is responsible for health parameter's sensing, the sink is responsible for data aggregation from all sensors, fault tolerance, and outside communication, while the mobile device is responsible for data processing, calibration and presentation. As a result, if the mobile device malfunctions or is not present, data is not compromised.

In Phone -Centered Body Sensor Network [9] Platform consists of one mobile phone, multiple sensor nodes, and a Cache-watch Sensor nodes and the Cache-watch communicate directly with the phone via Bluetooth. With limitation of battery life, user acceptance, storage, with Bluetooth imposing power overhead along with minimum range.

A Wireless Body Sensor Network (WBSN) for healthcare monitoring is presented in [10], where a sink and a central 
system are used. The sink acts as the "middleman" between the WBSN and the central system. The central system is a standard personal computer and is intended for hospital facilities rather than for personal use.

Another solution to the growing problem of older adults care comes from [11]. The authors present the telecare concept and provide some information about a pilot study. The need for thresholds to achieve flexibility and support a broad range of individuals is also mentioned.

Several approaches exist with different design goals, but the same purpose: to provide health information to the patient, to the medical staff or for both. In this work we aim to provide a convenient mean to sense bio-signals, process it and show health information. Since nowadays almost everyone carries around a mobile phone, the choice seems obvious.

\section{SYSTEM ARCHITECTURE}

The proposed wireless body sensor network for health monitoring integrated into a three tier telemedicine system. The lowest level consist set of intelligent sensors or nodes. These are the reduced function devise. The second level is the personal server (Internet enabled PDA, cell-phone, or home computer). These are full function devices. The third level encompasses a network of remote server which is the remote application to which data or information is transferred.

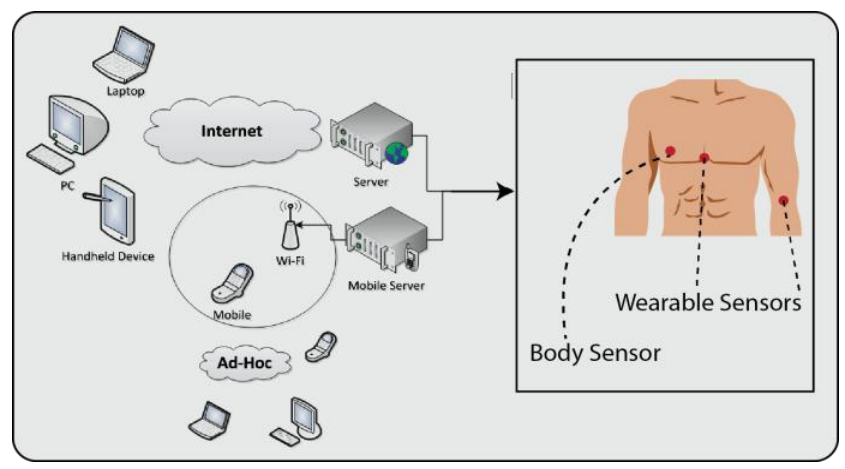

Figure 1: System Architecture

\section{Operating Environment}

Micro-controllers are useful to the extent that they communicate with other devices, such as sensors, motors, switches, keypads, displays, memory and even other microcontrollers.

The microcontroller board chosen for this project is AT89S52 is a low-power, high-performance CMOS 8-bit microcontroller with $8 \mathrm{~K}$ bytes of in-system programmable Flash memory. The AT89S52 provides the following standard features: $8 \mathrm{~K}$ bytes of Flash, 256 bytes of RAM, 32 I/O lines, Watchdog timer, two data pointers, three 16-bit timer/counters, a six-vector two-level interrupt architecture, a full duplex serial port, on-chip oscillator, and clock circuitry.

The microcontroller [1] is interfaced to the laptop (server) through serial port communication. In this system, we used a 9600 baud connection with 8 data bits, no parity bit, and one stop bit.With this configuration, data can be sent over RF wireless communication.

Keil [8] is an IDE (Integrated Development Environment) which is used to develop an application program, compile and run it even the code can be debugged .It is a simulator where we can check the application code even in the absence of the hardware board.
Keil is also a cross compiler. The process of development of the soft code on a processor for a particular application and which can be implemented on the target processor is known as Cross Development. In our design the main heart of the hardware module is the micro controller which is the programmable IC .The programming language used for developing the software to the micro controller is Embedded C. The KEIL cross compiler is used to edit, compile and debug this program. Programmer is used for burning the developed code on Keil into the micro controller Chip.

\section{Sensors}

Wearable sensors and systems have evolved to the point that they can be considered ready for clinical application. The use of wearable monitoring devices that allow continuous or intermittent monitoring of physiological signals is critical for the advancement of both the diagnosis as well as treatment of diseases.

Wearable systems are totally non-obtrusive devices that allow physicians to overcome the limitations of ambulatory technology and provide a response to the need for monitoring individual's over weeks or months.

\section{Heart beat sensor}

For heart beat sensor [7] each time the heart muscle contracts. Blood is ejected from the ventricles and a pulse of pressure is transmitted through a circulated system. This pressure pulse when traveling through the vessels causes vessel wall displacement which is measurable at various points. In order to detect pulsatile blood volume changes by photo electric method, photo conductors are used. Normally photo resistors are used, for amplification purpose photo transistors are used.

Light is emitted by LED and transmitted through the artery and the resistance of photo resistor is determined by the amount of light reaching it. With each contraction of heart, blood is forced to the extremities and the volume of blood in the finger increases, it alters the optical density with the result that the light transmission through the finger reduces and the resistance of the photo resistor increases accordingly. The photo resistor is connected as a part of voltage divider circuit and produces a voltage that varies with the amount of blood in the finger.

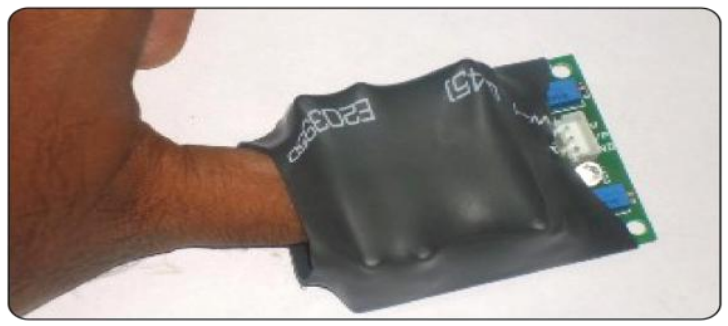

Figure 2: Pulse sensor

\section{Temperature sensor}

Due to unavailability of body temperature monitoring sensor during our experimentation we have utilized environmental temperature monitoring sensor [LM35].

The LM35 [6] is a precision integrated-circuit temperature sensor, whose output voltage is linearly proportional to the Celsius (Centigrade) temperature. The LM385 is a voltage regulator diode. Analog to digital converter (ADC) is used for converting analog value got as input. ADC08031 is used as ADC, which gives a digital value with a serial output with 
digital temperature transmitter circuit. Its full-scale is up to +128 deg C. The output can be taken out by the following pins: CLK, ENA, respectively enable client-side and the clock as shown in figure 3 .

These sensors use a solid-state technique to determine the temperature. They use the fact as temperature increases, the voltage across a diode increases at a known rate. (Technically, this is actually the voltage drop between the base and emitter the Vbe - of a transistor. By precisely amplifying the voltage change, it is easy to generate an analog signal that is directly proportional to temperature.

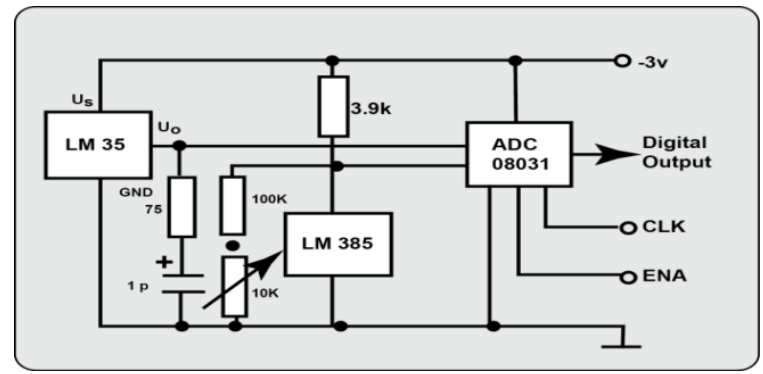

Figure 3: Voltage output with integrated circuit Temperature sensor LM35

\section{MAX232}

The MAX232 [8] is an integrated circuit that converts signals from an RS-232 serial port to signals suitable for use in TTL compatible digital logic circuits. The MAX232 is a dual driver/receiver and typically converts the RX, TX, CTS and RTS signals.

The drivers provide RS-232 voltage level outputs (approx. $\pm 7.5 \mathrm{~V}$ ) from a single $+5 \mathrm{~V}$ supply via on-chip charge pumps and external capacitors. This makes it useful for implementing RS-232 in devices that otherwise do not need any voltages outside the $0 \mathrm{~V}$ to $+5 \mathrm{~V}$ range, as power supply design does not need to be made more complicated just for driving the RS-232 in this case.

The receivers reduce RS-232 inputs (which may be as high as $\pm 25 \mathrm{~V}$ ), to standard $5 \mathrm{~V}$ TTL levels. These receivers have a typical threshold of $1.3 \mathrm{~V}$, and a typical hysteresis of $0.5 \mathrm{~V}$.

\section{Microsoft Visual Studio}

Microsoft Visual Studio [8] is an integrated development environment (IDE) from Microsoft. It can be used to develop graphical user interface [GUI] applications supported by Microsoft Windows, Windows Mobile, Windows CE, .NET Framework, .NET Compact Framework.

The GUI is created using Visual C\# studio 2005, to display the measured temperature and pulse of the patient. The code written also determines the abnormalities of temperature and heart beat of a patient.

\section{MSSQL}

MSSQL 2005 is used for storing biosignals sensed by the sensors. After a body sensor generates a measurement, it transmits sensed data to the base station. The base station (used here is personal computer) can be located within radio range of sensors. After data cleaning and summarizing, it is stored in the database (MSSQL) of the base station. The database will store all kinds of data such as sensed body/environment/ location/time data.

The doctor can check the patient condition by accessing the data from the server via the interface provided to him. Here the base station itself act as server for receiving data from sensor and sending to caretaker or for remote monitoring by doctors.

\section{SIMULATION RESULTS}

\subsection{Serial Port Communication}

The .NET framework version 2.0 (beta) provides features for serial communication. The framework provides System.IO.Ports namespace. The new framework provides classes with the ability to access the serial ports on computer, and to communicate with serial I/O devices. We are using RS $232 \mathrm{C}$ standard for communication between PCs.

Initially port number and other properties are fetched from application configuration file to allow the user to set the appropriate properties ( i.e., Instantiate the communications port with data stored in application configuration file settings)

Properties are set as follows:

$$
\begin{aligned}
& \text { "Port" value="COM4", "BaudRate" value }=" 9600 " \\
& \text { "Parity" value="None", "DataBits" value }=" 8 "
\end{aligned}
$$

The read timeout is set to 1000 milliseconds and null bytes are ignored when transmitted between the port and the receive buffer. String using the SerialPort.ReadExisting() method is used to read data into a byte buffer and generate an event when you detect a valid packet in the data. And store data into string variable.

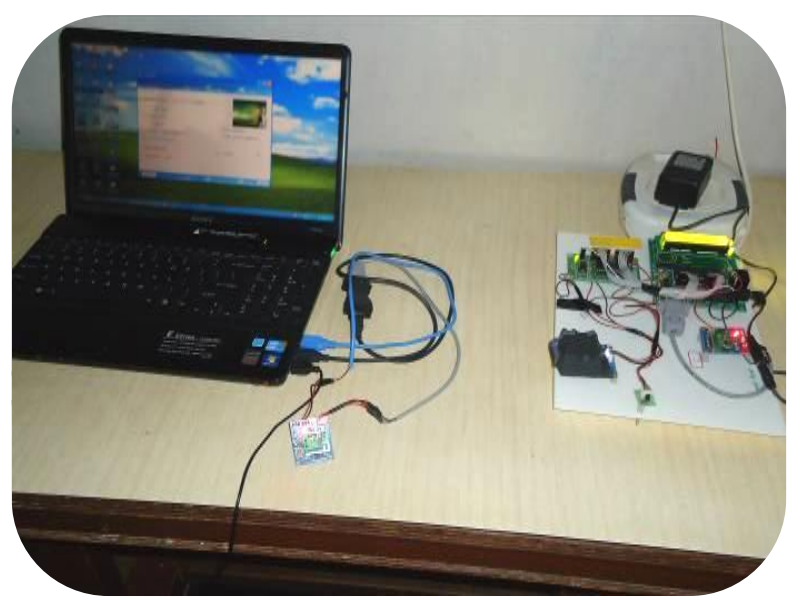

Figure 4: Overview of proposed model

The proposed system is used for monitoring the heart rate of patient and temperature; output will be two parameters (Heart rate and Temperature). The data that has been received from the serial port is then analyzed and configured to the desired format to display in the User Interface. The incoming data is in the Temperature, pulse rate format, e.g.: 035072. Where data is split and displayed in desired format by editor. The \# is used as delimiter to separate the two reading of body temperature and pulse rate.

The GUI of the presented system is as shown in figure 5. 


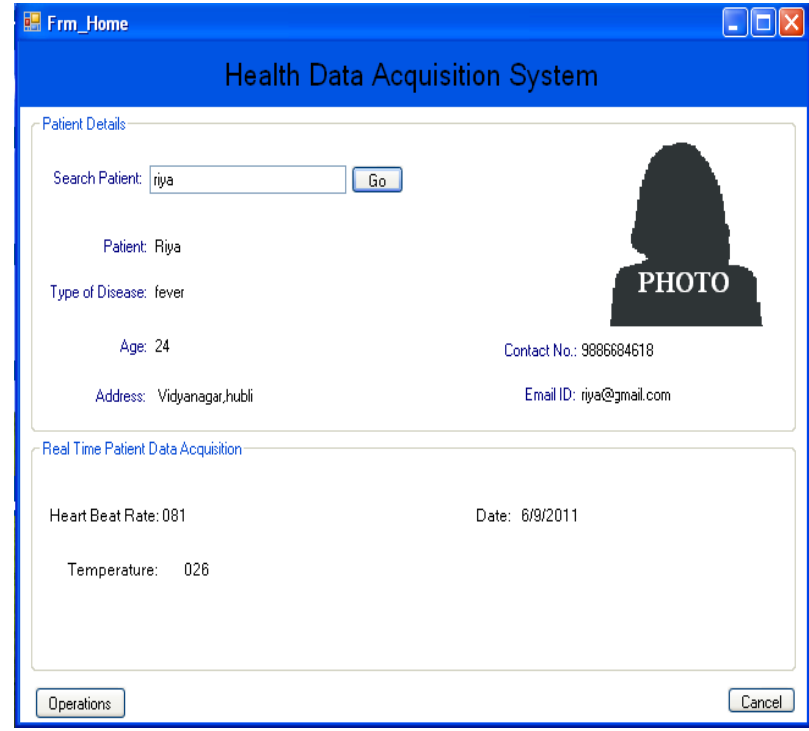

Figure 5: User interface for proposed system

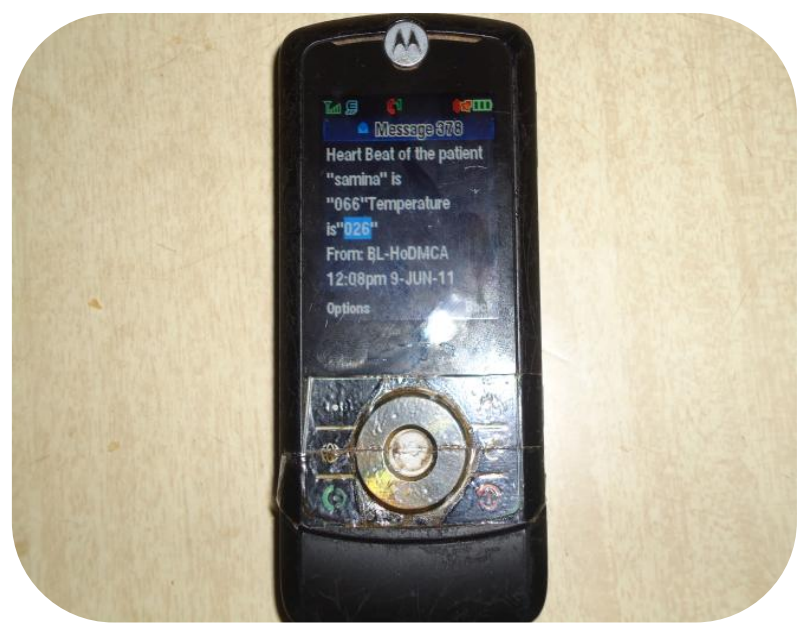

Figure 6: SMS sent to patient or caretaker mobile

\section{Fuzzy LoGIC}

Fuzzy-logic theory [4] has been mainly applied to industrial problems including production systems. There has been significant attention given to modeling scheduling problems within a fuzzy framework. Several fuzzy logic based scheduling systems have been developed, although direct comparisons between them are difficult due to their different implementations and objectives. In general, a Fuzzy Logic System (FLS) is a nonlinear mapping of an input data vector into a scalar output. Figure 7 depicts a FLS that is widely used in fuzzy logic controllers. A FLS maps crisp inputs into crisp outputs, and this mapping can be expressed quantitatively as y $=f(x)$. It contains four components: fuzzifier, fuzzy rules, inference engine, and defuzzifier.

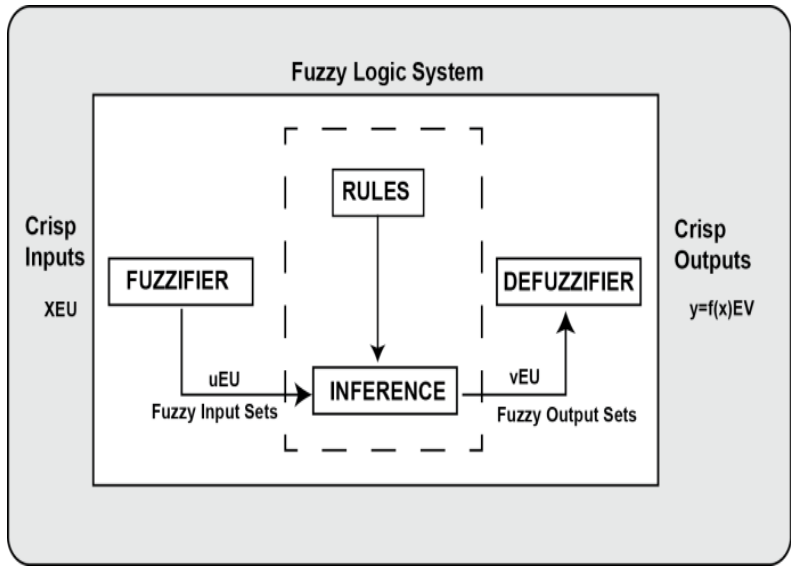

Figure 7: The structure of FLC system

Here, we have designed a FLC system for health monitoring services, which is one of component in our pervasive computing prototype health status. The FLC system receives context information from sensor (sensor data stored in data base) equipments as the inputs of the FLC and the fuzzification module converts inputs into fuzzy linguistic variable inputs.

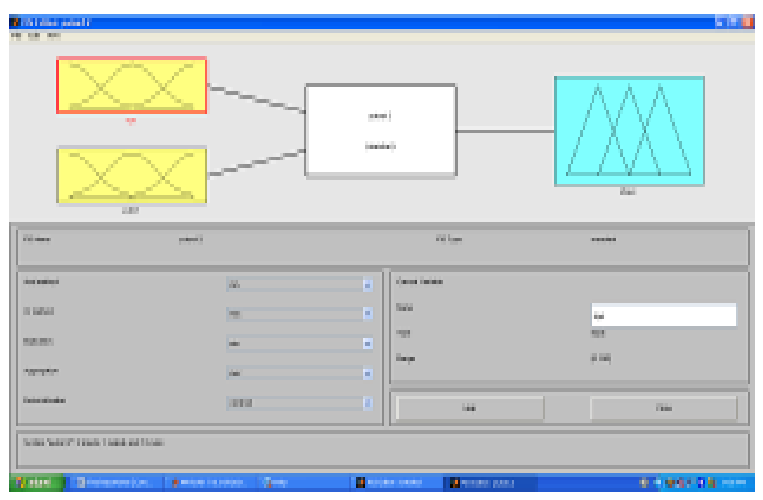

Figure 8: FIS editor

On analyzing the data requirements of health services, four linguistic variables were defined, representing the physical sign of patient. The membership functions of these input parameters of the fuzzy logic are illustrated in Fig.9. The labels in the fuzzy variables are presented as follows.

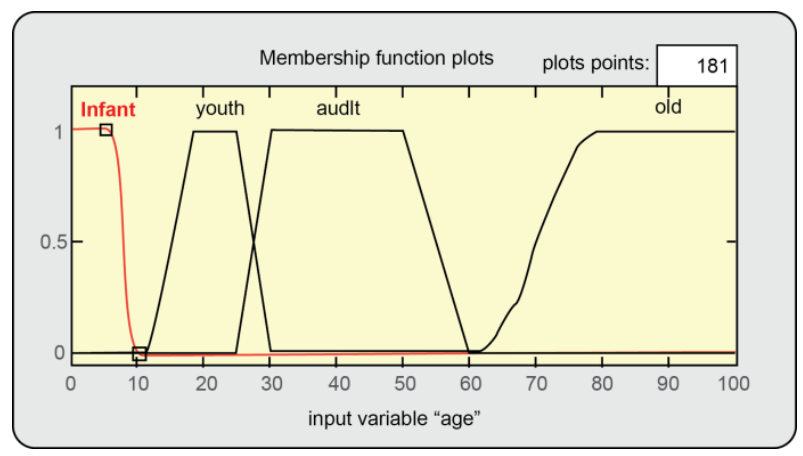

Figure 9: Membership functions for input age Age $=\{$ infant, youth, adult, old $\}$ 


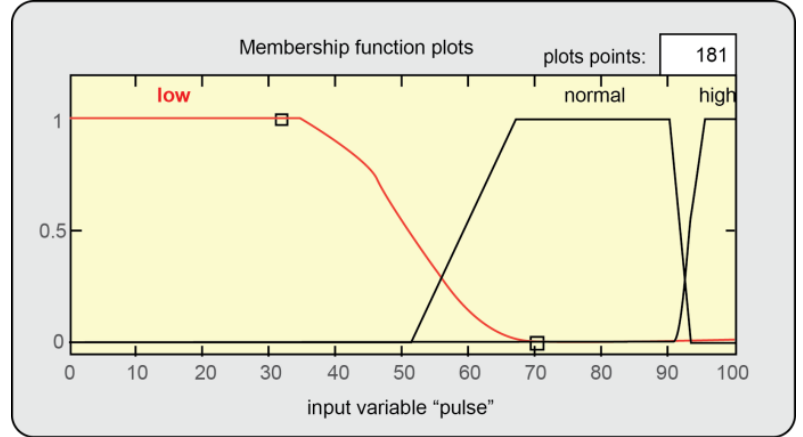

Figure 10: Membership functions for input pulse Pulse $=\{$ low, normal, critical $\}$;

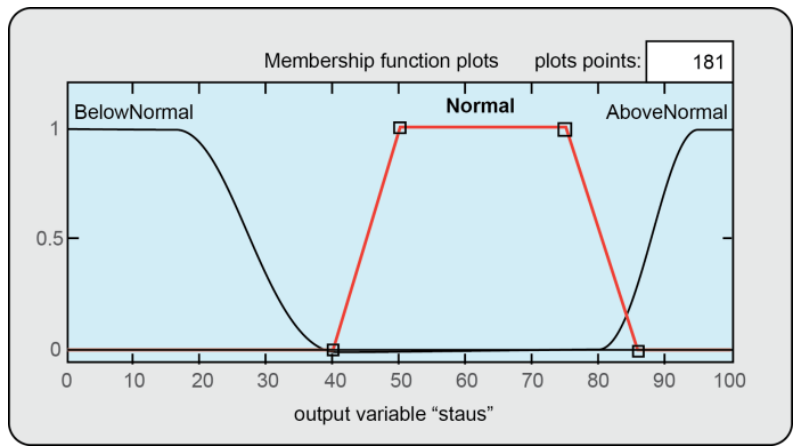

Figure 11: Membership functions for output status

Status $=\{$ BelowNormal, Normal, AboveNormal $\} ;$

Status values are evaluated based on rules given below:

If (Age is Infant) and (Pulse is Normal) then Status is Normal

If (Age is Infant) and (Pulse is High) then Status is Normal

If (Age is Youth) and (Pulse is High) then Status is Above Normal

If (Age is Youth) and (Pulse is Low) then Status is Below Normal.

A sample fuzzy calculation at a value of context information point is described in Figure 12.

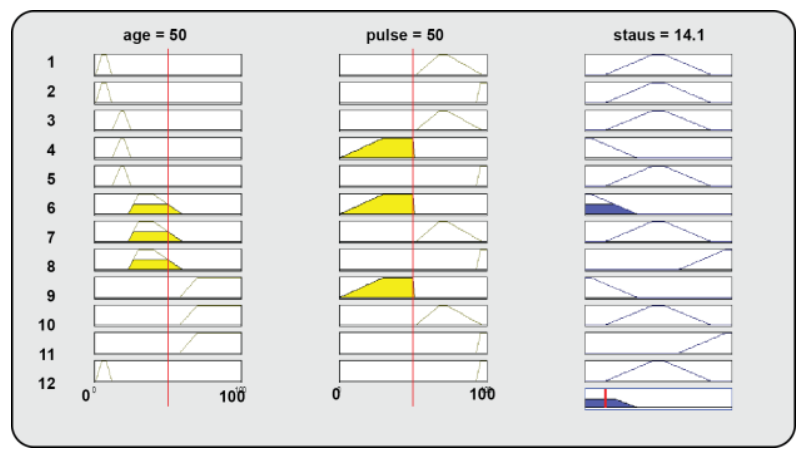

Figure 12: Sample fuzzy calculation

To calculate status values for all the health data entries from database, first database connectivity is done using function "conn()" here default mssql server connection is made where the health data is stored using serial port communication. After fetching the data from database using "fetch()" function for fuzzfication it is evaluated using evalfis() and displayed. The final output is stored into text file with first column input pulse, second column as defuzzified output and third column as status as shown in figure 12 .

conn = database ('.,',",");

curs=exec(conn, 'SELECT DISTINCT HBeatRate,

Age FROM Demo1.dbo.StatusData');

Out $=$ evalfis $(t, b)$;

Status values are evaluated for all the health data entries stored in database. The status value is to be evaluated based on both age and pulse sensed. Here, status is evaluated by sensing the pulse value for different age groups. Keeping age as constant and sensed pulse sensor is attached to one person at a time. In the figure 12 shows defuzzified value for patient with age 32 , where variation is shown in status/output if only pulse is considered as input, this is shown in figure 14 where the first graph is taken by considering pulse as only input to evaluate status of patient whereas graph second considers both age and pulse as input parameter as a result there is variation in the output (i.e. graph). As in case of infant, if the pulse of new born is 95 then it is considered as normal with respect to age, if only pulse is taken as output without age then status may be wrong in some cases.I

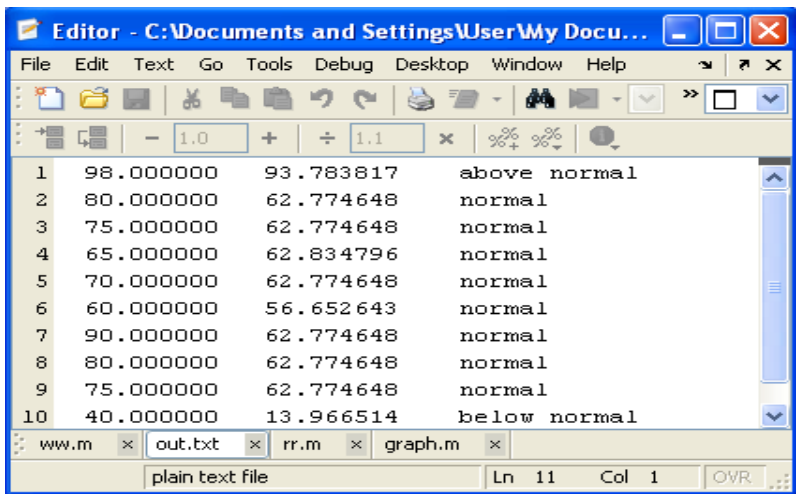

Figure 13: Output text file

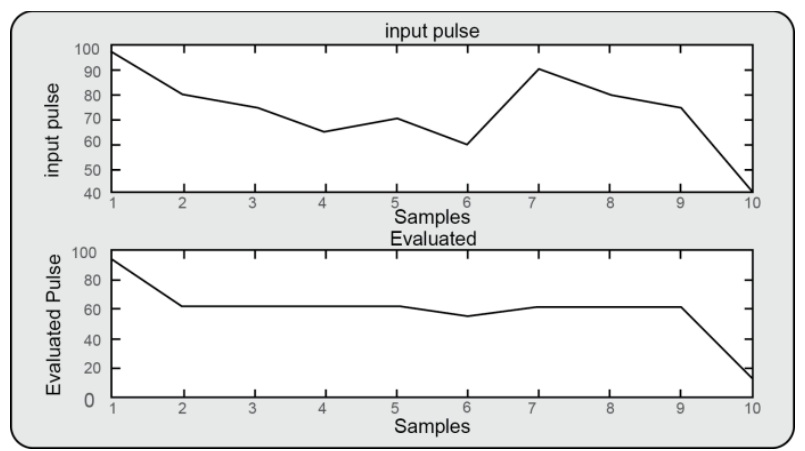

Figure 14: Plot for Input and Evaluated Pulse

\section{CONCLUSION}

This paper demonstrates the use of wearable and implantable Wireless Body Sensor Network as a key infrastructure enabling unobtrusive, constant, and ambulatory health monitoring. This new technology has potential to tender a wide range of assistance to patients, medical personnel, and society through continuous monitoring in the ambulatory environment, early detection of abnormal conditions, 
supervised restoration, and potential knowledge discovery through data mining of all gathered information.

In this work, we have analyzed the on-line health monitoring system of temperature and pulse of patient using SMS and feedback/prescription from doctor given through mail. Any abnormalities in health conditions are informed via SMS to the doctor/emergency ambulance. Our study in this paper also demonstrates that fuzzy logic can be applied to health monitoring system using wireless sensor network for deciding final observation of patient health status based on bio-signal reading.

This paper proves that wireless sensor networks can be widely used in healthcare applications. We believe that the role of Body sensor networks in medicine can be further enlarged and we are expecting to have a feasible and proactive prototype for wearable / implantable WBSN system, which could improve the quality of life.

\section{ACKNOWLEDGMENT}

The authors would like to thank Dr. Ashok Shettar, Principal and Prof. B.L.Desai Vice Principal, B.V.B College of Engg., $\&$ Tech for their valuable support and motivation for our work

\section{REFERENCES}

[1] Brad R. Geltz, Jacob A. Berlier, James M. McCollum, Ph.D. "Using the iPhone and iPod Touch for Remote Sensor Control and Data Acquisition", IEEE 2010.

[2] Josephine Selvarani, "ONLINE HEALTH MONITORING SYSTEM USING ", presented publication in International Journal on Computer Science and Engineering" Karunya University, Coimbatore, 2011

[3] Orlando R. E. Pereira, Paulo A. C. S. Neves, Joel J. P. C. Rodrigues, "Mobile Solution for Three-tier Biofeedback
Data Acquisition and Processing ", presented publication in the IEEE "GLOBECOM" proceedings, Portugal,2008.

[4] Begonya Otal, Luis Alonso and Christos Verikoukis, "A new MAC Approach in Wireless Body Sensor Networks for Health Care ", presented publication in the IEEE "GLOBECOM" proceedings, Portugal,2008

[5] Begonya Otal, Luis Alonso and Christos Verikoukis, A new MAC Approach in Wireless Body Sensor Networks for Health Care, 2nd ed., R. M. Osgood, Jr., Ed. Berlin, Germany: Springer-Verlag, 1998.

[6] http://www.nskelectronics.com/files/lm35.pdf

[7] Heartbeat sensor , Available: http:// http://www.nskelectronics.com/heart_beat.html

[8] Sana Ullah, Pervez Khan et.al , A Review of Wireless Body Area Networks

[9] A Review of Wireless Body Area Networks for Medical Applications, arXiv:1001.0831v3 [cs.NI] 3 Aug 2010

[10] L. Zhong, M. Sinclair, and R. Bittner, "A Phone-centered Body Sensor Network Platform Cost, Energy Efficiency \& User Interface", presented at International Workshop on Wearable and Implantable Body Sensor Networks (BSN 2006), Cambridge, MA, USA, 2006

[11] S.-L. Chen, H.-Y. Lee, C.-A. Chen, C.-C. Lin, and C.-H. Luo, "A Wireless Body Sensor Network System for Healthcare Monitoring Application", presented at IEEE Biomedical Circuits and Systems Conference (BIOCAS 2007), Montréal, QC, Canada, 2007.

[12] A. A. Reeves, J. W. P. Ng, S. J. Brown, and N. M. Barnes, "Remotely Supporting Care Provision for Older Adults", presented at International Workshop on Wearable and Implantable Body Sensor Networks (BSN 2006), Cambridge, MA, USA, 2006. 